\title{
Contenidos de isómeros trans de los ácidos grasos en productos cárnicos. (III) Tejido adiposo y grasa intramuscular de vacuno
}

\author{
Por M.C. Lluch, M. Roca de Vinyals, J. Parcerisa, F. Guardiola, R. Codony, M. Rafecas y J. Boatella \\ Departamento de Ciencias Fisiológicas Humanas y de la Nutrición. \\ Unidad de Nutrición y Bromatología. Universidad de Barcelona.
}

\section{RESUMEN}

Contenidos de isómeros trans de los ácidos grasos en productos cárnicos. (III) Tejido adiposo y grasa intramuscular de vacuno.

Se presentan los resultados obtenidos para la determinación de ácidos grasos en una serie de muestras de tejidos subcutáneo y muscular, procedentes de canales de vacuno, por aplicación de la cromatografía en fase gaseosa, para los que se obtuvieron unos valores medios de $58.7 \%$ de ácidos saturados, $39.1 \%$ de monoinsaturados y $2.7 \%$ de polinsaturados, en el tejido adiposo, y de $44.7 \%$ de saturados, $46.1 \%$ de monoinsaturados y $9.4 \%$ de polinsaturados, en el tejido muscular. Los contenidos de ácidos grasos trans muestran diferencias significativas entre ambos tejidos (medias del $7 \%$ de ácidos trans totales en grasa intramuscular y $10.5 \%$ en grasa de depósito). El C18: 1t presenta una distribución paralela a la del total de ácidos trans, mientras que para el C16: 1 trans se observa un comportamiento claramente diferente, ya que no se presentan estas diferencias significativas entre ambos tejidos. En cuanto a los factores estudiados que pueden influir en el contenido de isómeros trans, cabe destacar que la raza fue aquél que ofrecía más diferencias, mientras que entre los diversos orígenes (explotaciones ganaderas) y entre categorías de canal se presentaron menos diferencias en relación a los contenidos de estos isómeros. También es importante destacar que las correlaciones que se han observado entre los contenidos de ácidos trans y los totales de ácidos saturados, mono y polinsaturados presentan un signo contrario, según el tipo de tejido. Así, un aumento del \% de ácidos trans va aparejado con un aumento de saturados y una disminución de polinsaturados en el tejido muscular, mientras que va aparejado con una disminución de saturados y un aumento de mono y polinsaturados en el tejido adiposo.

PALABRAS-CLAVE: Acido graso - Grasa intramuscular - Isómero trans (contenido) - Tejido adiposo - Vaca.

\section{SUMMARY}

Trans isomers contents of the fatty acids in meat products. (III) Adipose tissue and intramuscular fat from beef.

We give the results obtained for the determination of trans fatty acids in subcutaneous and intramuscular fats from different veal carcasses, using gas chromatography. FA composition differs clearly between both tissues, showing mean values of $58.7 \%$ SFA, $39.1 \%$ MUFA and $2.7 \%$ PUFA in the adipose tissue, and of $44.7 \%$ SFA, $46.1 \%$ MUFA and $9.4 \%$ PUFA in the muscle. Trans fatty acid values obtained in muscle and adipose tissue show statistically significant differences (mean values of $7 \%$ total trans fatty acids in intramuscular fat and $10.5 \%$ in adipose tissue fat). Besides, we observed a clearly different distribution for C 16: 1t, that didn't show these differences, while $\mathrm{C} 18: 1 \mathrm{t}$ showed a similar distribution than total trans fatty acids. Related to the factors that could determine the trans fatty acid contents in veal carcasses, we found that the race showed more differences between groups than the origin of the animal and their commercial category. On the other hand, correlations we found between trans fatty acids and the total of saturated, mono and polyunsaturated fatty acids showed an opposite sign according to the type of tissue (muscular or adipose). Thus, an increase in the trans fatty acid content was associated with a parallel increase of saturated and decrease in polyunsaturated fatty acid contents in the intramuscular fat. On the contrary, an increase of trans fatty acid content in adipose fat was correlated to a decrease of saturated and an increase of mono and polyunsaturated fatty acid contents.

KEY-WORDS: Adipose tissue - Beef - Fatty acid - Intramuscular fat Trans isomer (content).

\section{INTRODUCCION}

El presente trabajo es la continuación de dos anteriores (Hernández et al., 1991) (Lluch et al., 1993), en los que se abordaba la determinación del contenido de ácidos grasos trans en productos cárnicos y en la carne y grasa de cerdo, respectivamente. En el presente trabajo se establecen los niveles de ácidos trans en las canales de vacuno, así como su correlación con la composición lipídica en general y la influencia de factores genéticos y productivos, con el objetivo de conocer la variabilidad de los mismos y poder finalmente evaluar la ingesta de ácidos trans procedente de los productos de la carne. Cabe señalar que la bibliografía consultada no presenta datos de estos contenidos en canales procedentes de animales de nuestro país.

En relación a los principales factores que pueden condicionar el contenido de ácidos grasos trans en la carne de bovino cabe mencionar, en primer lugar, la transformación y síntesis de ácidos grasos que tiene lugar en el rumen, por acción de microorganismos específicos. Estos procesos implican, después de la hidrólisis de los glicéridos, una hidrogenación de dobles enlaces por vía microbiana, que aumenta la proporción de ácidos monoinsaturados y sobre todo de saturados (Harfoot, 1973) (Doreau et al., 1989) (Bauchart, 1981 ) (Davis, 1990), al mismo tiempo que se produce una isomerización parcial de los dobles enlaces restantes (Emken y Dutton, 1979) (Gunstone y Norris, 1983). La raza es, así mismo, otro factor de influencia, ya que determina un mayor o menor contenido graso de la canal (Girard et al., 1985). Diversos autores han descrito que esta variabilidad es mucho mayor para el bovino que para el cerdo y ovino (Bass et al., 1990) (Enser, 
1984). También es importante la localización anatómica y el tipo de tejido, que no sólo condiciona el contenido graso sino también su composición en ácidos grasos (Aurosseau, 1981 ) (Hecker et al., 1975) (Kinney et al., 1990). Finalmente, no cabe olvidar la importante participación del contenido de ácidos grasos trans en la alimentación del animal, que tendrá un efecto aditivo sobre los que ya se sintetizan por el propio animal. En este apartado cabe señalar que existen practicamente estudios sobre el efecto de la utilización de grasas «by-pass», de reciente introducción en la alimentación de los rumiantes, con la intención de protegerlas del efecto de hidrogenación y obtener así una grasa menos saturada (Gurr, 1984). Cabría esperar que este efecto de protección comportara también una menor formación de ácidos trans y, por ello, un menor contenido de estas formas en los productos con este origen, como son carnes, sebos, leche, mantequilla, nata (Sommerfeld, 1983) (Gurr, 1984).

Los estudios específicos sobre el contenido de ácidos grasos trans en la carne de vacuno y sus grasas son escasos (Lin et al., 1984) (Slover et al., 1987), y en la mayoría de los casos, los datos disponibles proceden de estudios globales de ingesta de ácidos trans (Akesson et al., 1981) (Hunter y Applewhite, 1986) (British Nutrition Foundation, 1987), en los que no se analizan aspectos relativos a la variabilidad.

\section{MATERIALES Y METODOS}

\subsection{Muestras}

Para este estudio se recogieron muestras de canales de ternera de diferentes mataderos de la provincia de Barcelona. Para cada muestra se confeccionó una ficha que recogía datos del origen (Zaragoza y cinco comarcas catalanas: Solsonés, Vallés, Osona, Bages y Urgell), sexo, raza (Charolesa, Asturiana y Frisona), peso y categoría ( $E, U$, $\mathrm{R}, \mathrm{O}$ ).

En el matadero, se tomó una muestra de $150 \mathrm{~g}$, aproximadamente, de músculo (Entrama o pilar del diafragma) y de $100 \mathrm{~g}$ de tejido graso perirrenal, de cada canal. Se obtu- vieron así un total de 86 muestras de cada tejido, que se conservaron a temperatura de congelación hasta el momento del análisis.

\subsection{Preparación de las muestras}

En las muestras de tejido muscular se eliminaron al máximo los restos de grasa intermuscular, de forma que se pudiese determinar así, exclusivamente, el contenido y composición de la grasa intramuscular. Las muestras de tejido adiposo se limpiaron, igualmente, con objeto de eliminar posibles restos de tejido cartilaginoso que lo pudieran acompañar.

En todos los casos, las muestras se sometieron a trituración hasta obtener una buena homogenización de los tejidos. Para cada determinación se tomaron dos alicuotas de cada muestra con el fin de asegurar la representatividad de los resultados.

\subsection{Métodos analíticos}

a) Extracción de la grasa: Para todas las muestras se ha utilizado el método propuesto por Folch et al. (1957).

b) Determinación de la composición en ácidos grasos por cromatografía en fase gaseosa: La preparación de los ésteres metílicos se realizó de acuerdo con el método propuesto por Slover y Lanza (1979), que utiliza $\mathrm{BF}_{3} /$ Metanol como reactivo y hexano como disolvente de extracción. EI análisis cromatográfico se ha llevado a cabo en un cromatógrafo Perkin Elmer Sigma 2000, acoplado a un integrador microordenador PE Nelson 1020 y equipado con una columna capilar tipo WCOT de sílice fundida de $50 \mathrm{~m}$ de largo y $0.25 \mathrm{~mm}$ de diámetro interno, recubierta con fase estacionaria líquida de $100 \%$ cianopropilpolisiloxano (CP Sil 88, Chrompack). Las condiciones cromatográficas fueron las siguientes: Temperatura del horno isoterma a $190^{\circ} \mathrm{C}$, del inyector a $300^{\circ} \mathrm{C}$ y del detector a $270^{\circ} \mathrm{C}$. Como gas portador se utilizó Helio, a una presión de entrada de 15 psi y con una relación de split 1:100.

Para la identificación se han utilizado patrones de los

Tabla I

Contenidos de ácidos grasos trans y composición lipídica simplificada para el conjunto total de las muestras

\begin{tabular}{|c|c|c|c|c|c|c|c|}
\hline & $\%$ grasa & C16:1 t & C18:1 t & Total t & Sat & Mono & Poli \\
\hline \multicolumn{8}{|c|}{ Tejido adiposo } \\
\hline$x$ & - & 0,9 & 9,6 & 10,5 & 58,7 & 39,1 & 2,7 \\
\hline DE & - & 0,1 & 3,1 & 3,2 & 4,3 & 4,3 & 0,9 \\
\hline $\max$ & - & 1,3 & 20 & 21,3 & 69,1 & 52,4 & 5,5 \\
\hline $\min$ & - & 0,5 & 2,1 & 3,1 & 46,4 & 27,7 & 0,8 \\
\hline $\mathrm{n}$ & - & 77 & 77 & 77 & 77 & 77 & 77 \\
\hline \multicolumn{8}{|c|}{ Tejido muscular } \\
\hline$x$ & 3,6 & 1,1 & 5,9 & 7 & 44,7 & 46,1 & 9,4 \\
\hline $\mathrm{DE}$ & 2 & 1,1 & 3 & 3 & 4,7 & 4,4 & 4,4 \\
\hline $\max$ & 9,8 & 8,3 & 12 & 13,9 & 54,7 & 57,2 & 23,4 \\
\hline $\min$ & 0,5 & 0 & 0,2 & 0,3 & 34,5 & 39,2 & 2 \\
\hline $\mathrm{n}$ & 86 & 86 & 86 & 86 & 86 & 86 & 86 \\
\hline
\end{tabular}

Resultados de AG expresados como normalización interna. 
Tabla II

Contenidos de ácidos grasos trans en tejido adiposo y grasa intramuscular en función de la raza

\begin{tabular}{|c|c|c|c|c|c|c|c|c|c|}
\hline & \multicolumn{3}{|c|}{ Charolesa } & \multicolumn{3}{|c|}{ Asturiana } & \multicolumn{3}{|c|}{ Frisona } \\
\hline & C16:1t & C18:1 t & Total t & C16:1t & C18:1 t & Total t & $\mathrm{C} 16: 1 \mathrm{t}$ & C18:1 t & Total t \\
\hline Tejido adiposo & & & & & & & & & \\
\hline$x$ & 0,9 & 8,6 & 9,5 & 0,9 & 9,2 & 10,1 & 0,9 & 13,5 & 14,4 \\
\hline $\mathrm{DE}$ & 0,05 & 0,7 & 0,8 & 0,01 & 0,4 & 0,4 & 0,05 & 1,1 & 1,1 \\
\hline$n$ & 14 & 14 & 14 & 54 & 54 & 54 & 9 & 9 & 9 \\
\hline Tejido muscular & & & & & & & & & \\
\hline$x$ & 1,1 & 6,4 & 7,6 & 1 & 5,3 & 6,4 & 0,8 & 9 & 9,8 \\
\hline $\mathrm{DE}$ & 0,3 & 0,5 & 0,6 & 0,1 & 0,4 & 0,4 & 0,1 & 1,1 & 6,4 \\
\hline $\mathrm{n}$ & 23 & 23 & 23 & 52 & 52 & 52 & 9 & 9 & 9 \\
\hline
\end{tabular}

Resultados expresados como normalización interna.

diferentes ácidos grasos (Sigma). La cuantificación de los ácidos grasos se realizó por normalización interna.

\section{RESULTADOS Y DISCUSION}

\section{Resultados globales}

Las formas isoméricas que se detectan en este tipo de sustratos son únicamente $C 16: 1 t$ y $C 18: 1 t$, incluyéndose en ambos casos bajo esta denominación los diversos isómeros de posición. En la tabla I se exponen los valores medios y desviación estándar obtenidos, correspondientes a C16:1t, C18:1t y total de formas "trans", así como del total de ácidos saturados, monoinsaturados y polinsaturados en tejido adiposo y grasa intramuscular. Es importante señalar que para el caso del total de ácidos trans (medias del $7 \%$ en grasa intramuscular y $10.5 \%$ en tejido adiposo), se obtuvieron valores superiores a los publicados por otros autores (Slover et al. 1987) (Lin et al., 1984). Debe destacarse también que las diferencias observadas entre tejido adiposo y tejido muscular han sido estadísticamente significativas para los porcentajes relativos de formas trans totales $(P<0.0001$; intérvalo de confianza $=4.49-2.56)$ y de $C 18: 1 \mathrm{t}(\mathrm{P}<0.0001$; intérvalo de confianza $=4.63-2.74$ ), mientras que no se dieron estas diferencias significativas entre ambos tejidos para el $\mathrm{C} 16: 1 \mathrm{t}$.

\section{Influencia de la raza}

Por otra parte, en la Tabla II se recogen los valores medios correspondientes a los porcentajes relativos de C16:1t, C18:1t y total trans en tejido adiposo y grasa intramuscular, en función de la raza. El análisis de la varianza revela que existen diferencias significativas entre las medias de C18:1t y total trans (en ambos tejidos), en función de la raza, mientras que no existen diferencias para el C16:1t. Una prueba de Scheffé de contraste de hipótesis muestra que, en tejido adiposo, sólo existen diferencias entre las razas Frisona y Asturiana, para C18:1t (límites de confianza $=6.78-1.72$ ) y para el total trans (límites de confianza $=6.85-1.71$, así como entre las razas Frisona y
Charolesa, para C18:1t (límites de confianza $=7.88-1.88$ ) y para el total trans (límites de confianza $=7.97-1.87$ ). Para la grasa intramuscular, estas diferencias sólo se observaron entre las razas Frisona y Asturiana, para C18:1t (límites de confianza $=6.11-1.21$ ) y para el total trans (límites de confianza $=5.98-0.8$ ).

\section{Influencia de la categoría de la canal}

La Tabla III muestra los valores medios de contenidos de ácidos grasos trans en función de la categoría de la canal. El análisis de la varianza ha revelado la existencia de algunas diferencias significativas entre las medias correspondientes a las categorías contempladas, para el C18:1t $(P=0.0170)$ y el total de ácidos trans $(P=0.0166)$ en la grasa de depósito y sólo para el C18:1t intramuscular $(P=0.0421)$.

\section{Influencia del origen}

Así mismo, la Tabla IV recoge los contenidos medios de ácidos trans, en función del origen de los animales. La aplicación del análisis de la varianza muestra que no existen diferencias significativas para estos ácidos en la grasa intramuscular, mientras que existen diferencias, tanto para C16:1t ( $P=0.0028)$ y $C 18: 1 t(P=0.0009)$, como para el total de ácidos trans $(P=0.0007)$, en el caso del tejido adiposo.

\section{Correlaciones con otros componentes lipídicos}

Finalmente, se ha realizado un estudio de correlación entre variables, cuyos resultados se resumen en la tabla $\mathrm{V}$. Como primer comentario, cabe decir que se observa una correlación positiva entre el contenido graso intramuscular y el \% total de ácidos trans, así como entre dicho contenido graso y el \% C18:1t, mientras que, por el contrario, no existe correlación con el \% C16:1t. De la misma forma, se observa una correlación positiva del \% C18:1t y del \% de total trans con el \% de ácidos saturados en la grasa intramuscular, mientras que se presenta una correlación nega- 
Tabla III

Contenidos de ácidos grasos trans en tejido adiposo y muscular de ternera, en función de la categoría de la canal

\begin{tabular}{|c|c|c|c|c|c|c|}
\hline & \multicolumn{3}{|c|}{ Tejido adiposo } & \multicolumn{3}{|c|}{ Tejido muscular } \\
\hline & $\mathrm{C} 16: 1 \mathrm{t}$ & C18:1 t & Total t & $\mathrm{C} 16: 1 \mathrm{t}$ & $\mathrm{C} 18: 1 \mathrm{t}$ & Total $\mathrm{t}$ \\
\hline $\begin{array}{l}\text { Categoría E } \\
x \\
D E \\
n\end{array}$ & $\begin{array}{c}0,9 \\
0,02 \\
32\end{array}$ & $\begin{array}{c}9,8 \\
0,6 \\
32\end{array}$ & $\begin{array}{c}10,7 \\
0,6 \\
32\end{array}$ & $\begin{array}{c}1 \\
0,2 \\
32\end{array}$ & $\begin{array}{c}6,3 \\
0,4 \\
32\end{array}$ & $\begin{array}{l}7,3 \\
0,5 \\
32\end{array}$ \\
\hline $\begin{array}{l}\text { Categoría U } \\
x \\
D E \\
n\end{array}$ & $\begin{array}{c}0,88 \\
0,03 \\
14\end{array}$ & $\begin{array}{c}8 \\
0,7 \\
14\end{array}$ & $\begin{array}{c}8,9 \\
0,7 \\
14\end{array}$ & $\begin{array}{c}1,2 \\
0,2 \\
14\end{array}$ & $\begin{array}{c}4,2 \\
0,6 \\
14\end{array}$ & $\begin{array}{c}5,4 \\
0,7 \\
14\end{array}$ \\
\hline $\begin{array}{l}\text { Categoría R } \\
x \\
D E \\
n\end{array}$ & $\begin{array}{l}0,8 \\
0,02 \\
9\end{array}$ & $\begin{array}{l}8 \\
0,7 \\
9\end{array}$ & $\begin{array}{l}8,9 \\
0,7 \\
9\end{array}$ & $\begin{array}{l}1,13 \\
0,3 \\
9\end{array}$ & $\begin{array}{l}6,5 \\
0,8 \\
9\end{array}$ & $\begin{array}{l}7,6 \\
0,8 \\
9\end{array}$ \\
\hline $\begin{array}{l}\text { Categoría O } \\
x \\
D E \\
n\end{array}$ & $\begin{array}{c}0,9 \\
0,03 \\
20\end{array}$ & $\begin{array}{c}11 \\
0,8 \\
20\end{array}$ & $\begin{array}{r}11,9 \\
0,8 \\
20\end{array}$ & $\begin{array}{c}0,9 \\
0,2 \\
20\end{array}$ & $\begin{array}{c}6,8 \\
0,8 \\
20\end{array}$ & $\begin{array}{c}7,7 \\
0,8 \\
20\end{array}$ \\
\hline
\end{tabular}

Resultados expresados como normalización interna

tiva para los mismos ácidos trans, con el \% de ácidos polinsaturados. Por último, no se ha observado correlación entre el \% C16:1t y \% C18:1t, ni entre \% C16:1t y \% total de ácidos trans, mientras que sí existe correlación entre \% C18:1t y \% total de trans.
En relación a los contenidos de ácidos trans en tejido adiposo, cabe comentar que el estudio de correlación entre variables muestra, como hecho más significativo, que el C16:1t, que no mostraba ninguna correlación en el tejido intramuscular, sí que presenta correlaciones positivas con

Tabla IV

Contenidos de ácidos grasos trans en tejido adiposo y mu\$cular de ternera, en función del origen

\begin{tabular}{|c|c|c|c|c|c|c|}
\hline \multirow[b]{2}{*}{ Origen } & \multicolumn{3}{|c|}{ Tejido adiposo } & \multicolumn{3}{|c|}{ Tejido muscular } \\
\hline & $\mathrm{C} 16: 1 \mathrm{t}$ & $\mathrm{C} 18: 1 \mathrm{t}$ & Total $\mathrm{t}$ & $\mathrm{C} 16: 1 \mathrm{t}$ & $\mathrm{C} 18: 1 \mathrm{t}$ & Total $\mathrm{t}$ \\
\hline $\begin{array}{l}\text { Solsonés } \\
x \\
D E \\
n\end{array}$ & $\begin{array}{l}0,9 \\
0,05 \\
4\end{array}$ & $\begin{array}{l}8 \\
1 \\
4\end{array}$ & $\begin{array}{l}8,9 \\
1 \\
4 \\
\end{array}$ & $\begin{array}{l}0,8 \\
0,04 \\
4\end{array}$ & $\begin{array}{l}6 \\
0,7 \\
4\end{array}$ & $\begin{array}{l}6,8 \\
0,7 \\
4 \\
\end{array}$ \\
\hline $\begin{array}{l}\text { Vallés } \\
x \\
\text { DE } \\
n\end{array}$ & $\begin{array}{l}1 \\
0,04 \\
5 \\
\end{array}$ & $\begin{array}{c}10,2 \\
0,8 \\
5 \\
\end{array}$ & $\begin{array}{c}11,3 \\
0,8 \\
5 \\
\end{array}$ & $\begin{array}{l}0,8 \\
0,03 \\
5 \\
\end{array}$ & $\begin{array}{l}5,8 \\
0,5 \\
5 \\
\end{array}$ & $\begin{array}{l}6,6 \\
0,6 \\
5 \\
\end{array}$ \\
\hline $\begin{array}{l}\text { Osona } \\
x \\
D E \\
n\end{array}$ & $\begin{array}{c}0,9 \\
0,02 \\
24\end{array}$ & $\begin{array}{l}8,2 \\
0,6 \\
24\end{array}$ & $\begin{array}{l}9,1 \\
0,6 \\
24\end{array}$ & $\begin{array}{l}1,4 \\
0,2 \\
24 \\
\end{array}$ & $\begin{array}{l}5,1 \\
0,5 \\
24\end{array}$ & $\begin{array}{l}6,5 \\
0,5 \\
24 \\
\end{array}$ \\
\hline $\begin{array}{l}\text { Bages } \\
x \\
D E \\
n\end{array}$ & $\begin{array}{l}1 \\
0,1 \\
6\end{array}$ & $\begin{array}{c}13,9 \\
1,5 \\
6\end{array}$ & $\begin{array}{c}14,8 \\
1,6 \\
6\end{array}$ & $\begin{array}{l}0,8 \\
0,1 \\
6\end{array}$ & $\begin{array}{l}8,9 \\
1,7 \\
6\end{array}$ & $\begin{array}{l}9,7 \\
1,7 \\
6\end{array}$ \\
\hline $\begin{array}{l}\text { Urgell } \\
x \\
D E \\
n\end{array}$ & $\begin{array}{c}0,9 \\
0,02 \\
32 \\
\end{array}$ & $\begin{array}{r}10,2 \\
0,5 \\
32\end{array}$ & $\begin{array}{c}11,1 \\
0,5 \\
32 \\
\end{array}$ & $\begin{array}{c}1 \\
0,2 \\
32 \\
\end{array}$ & $\begin{array}{c}6 \\
0,5 \\
32 \\
\end{array}$ & $\begin{array}{c}7 \\
0,6 \\
32 \\
\end{array}$ \\
\hline $\begin{array}{l}\text { Zaragoza } \\
x \\
D E \\
n\end{array}$ & $\begin{array}{l}0,8 \\
0,1 \\
6\end{array}$ & $\begin{array}{l}8,3 \\
1,5 \\
6\end{array}$ & $\begin{array}{l}9,1 \\
1,5 \\
6\end{array}$ & $\begin{array}{l}0,8 \\
0,03 \\
6\end{array}$ & $\begin{array}{l}7,1 \\
1,1 \\
6\end{array}$ & $\begin{array}{l}7,8 \\
1,1 \\
6\end{array}$ \\
\hline
\end{tabular}


Tabla V

Correlaciones $(n=44)$ entre porcentajes de ácidos grasos trans en grasa de depósito e intramuscular y con otros parámetros lipídicos

\begin{tabular}{|c|c|c|c|c|c|c|}
\hline & C16:1 t & C18:1 t & Sat & Mono & Poli & \% Lípidos \\
\hline & \multicolumn{6}{|c|}{ Tejido muscular } \\
\hline C16:1t & & & $\begin{array}{c}\mathrm{r}=0,1293 \\
\text { NS }\end{array}$ & $\begin{array}{c}r=-0,0076 \\
N S\end{array}$ & $\begin{array}{c}r=-0,1617 \\
\text { NS }\end{array}$ & $\begin{array}{c}r=-0,1133 \\
\text { NS }\end{array}$ \\
\hline $\mathrm{C} 18: 1 \mathrm{t}$ & $\begin{array}{c}r=0,0252 \\
\text { NS }\end{array}$ & & $\begin{array}{l}r=0,4348 \\
p=0,0032\end{array}$ & $\begin{array}{c}r=-0,1180 \\
N S\end{array}$ & $\begin{array}{l}r=-0,3329 \\
p=0,0273\end{array}$ & $\begin{array}{l}r=0,4787 \\
p=0,0010\end{array}$ \\
\hline \multirow[t]{2}{*}{ Total t } & $\begin{array}{c}r=0,1885 \\
\text { NS }\end{array}$ & $\begin{array}{l}r=0,9865 \\
p=0,0000\end{array}$ & $\begin{array}{l}r=0,4483 \\
p=0,0023\end{array}$ & $\begin{array}{c}r=-0,1172 \\
N S\end{array}$ & $\begin{array}{l}r=-0,3535 \\
p=0,0186\end{array}$ & $\begin{array}{l}r=0,4517 \\
p=0,0021\end{array}$ \\
\hline & \multicolumn{6}{|c|}{ Tejido adiposo } \\
\hline $\mathrm{C} 16: 1 \mathrm{t}$ & & & $\begin{array}{l}r=0,3033 \\
p=0,0453\end{array}$ & $\begin{array}{c}r=0,0680 \\
\text { NS }\end{array}$ & $\begin{array}{l}r=0,3675 \\
p=0,0141\end{array}$ & \\
\hline $\mathrm{C} 18: 1 \mathrm{t}$ & $\begin{array}{l}r=0,2984 \\
p=0,0492\end{array}$ & & $\begin{array}{l}r=-0,3408 \\
p=0,0236\end{array}$ & $\begin{array}{l}r=0,6386 \\
p=0,0000\end{array}$ & $\begin{array}{l}r=0,5657 \\
p=0,0001\end{array}$ & \\
\hline Total t & $\begin{array}{l}r=0,3298 \\
p=0,0288\end{array}$ & $\begin{array}{l}r=0,9995 \\
p=0,0000\end{array}$ & $\begin{array}{l}r=-0,3266 \\
p=0,0305\end{array}$ & $\begin{array}{l}r=0,6340 \\
p=0,0000\end{array}$ & $\begin{array}{l}r=0,5722 \\
p=0,0000\end{array}$ & \\
\hline
\end{tabular}

Resultados de AG expresados como normalización interna.

$\mathrm{NS}=$ no significativo.

el \% C18:1t, con el \% total de ácido trans y con el \% de ácidos saturados, mientras que presenta una correlación inversa con el \% de polinsaturados. Respecto a los \% de C18:1t y de trans totales, cabe destacar que se detecta una buena correlación con el \% de ácidos monoinsaturados, cosa que no sucedía para la grasa intramuscular. lgualmente, es importante destacar que las correlaciones que presentan C18:1t y los ácidos trans totales con el \% de saturados y con el \% de polinsaturados tienen carácter inverso a las que se presentaban en la grasa intramuscular. Es decir que, mientras que los valores de ácidos trans aumentan con el \% de saturados y disminuyen con el $\%$ de polinsaturados en la grasa muscular, en el tejido adiposo sucede exactamente lo contrario.

\section{CONCLUSIONES}

Los contenidos (\% relativos) de isómeros trans en tejido adiposo y grasa intramuscular de ternera se sitúan, para las muestras estudiadas, dentro de márgenes que podrían considerarse bastante elevados, respecto a los valores medios publicados por otro autores (Slover et al., 1987) (Lin et al., 1984). Este hecho puede deberse a una influencia de la alimentación del animal y, más concretamente, al tipo de grasas (fundamentalmente sebos) utilizadas en la elaboración de piensos. Confirman esta hipótesis las diferencias significativas observadas entre valores medios de formas trans en tejido adiposo, en función del origen y que no se ponen de manifiesto en función de la raza.

Por otra parte, debe destacarse el hecho de que la variabilidad observada para el contenido de $\mathrm{C} 16: 1 \mathrm{t}$ sea mucho menor que la de C18:1t y la del total de formas trans en tejido adiposo. Ello parece explicable dado que, por ser compartimento de depósito, refleja más directamente la composición en ácidos grasos procedentes de grasas de la dieta, modificadas además por el proceso de biohidrogenación que afecta especialmente a la fracción C18.

Al mismo tiempo, en las muestras estudiadas se han observado contenidos significativamente mayores para el total de formas trans y de C18:1t para la grasa intramuscular, en comparación con la de depósito, hecho que no se presenta para el C16:1t. Ello indica la existencia de factores endógenos que conducen a una diferente utilización metabólica de estos ácidos trans en ambos tipos de tejidos.

Del estudio global de correlaciones, debe destacarse el hecho de que mayores contenidos de grasa intramuscular se corresponden con porcentajes más elevados de formas C18:1t. Este fenómeno se ha observado también en un estudio similar realizado con muestras de cerdo (Lluch et al., 1993). En este mismo sentido, cabe señalar la existencia de una relación positiva entre C18:1t y ácidos saturados y negativa con los polinsaturados, en tejido muscular. Este hecho podía confirmar la hipótesis de utilización de formas trans en procesos de síntesis sugerida por Lands y colaboradores (1966). Sin embargo, en el caso del tejido adiposo, estas correlaciones cambian de signo, ya que, a mayor insaturación se observan porcentajes más elevados de formas trans, posiblemente como consecuencia de la influencia de la alimentación sobre la composición de este tejido.

Finalmente, debe destacarse que entre los factores de variabilidad estudiados, la raza es el que ofrece mayores diferencias de contenidos trans en grasa intramuscular, mientras que raza y origen son principales responsables de la variabilidad del tejido adiposo.

\section{AGRADECIMIENTOS}

Este trabajo ha sido posible gracias al disfrute de una «Ayuda a la investigación» de la Caixa de Barcelona. 


\section{BIBLIOGRAFIA}

Akesson, B., Johansson, B.M., Svensson, M., Ockerman, P.A. (1981)-“Content of trans-octadecenoic acid in vegetarian and normal diets in Sweden, analyzed by the duplicate portion technique".- Am. J. Clin. Nutr. 34, 2517-2520.

Aurousseau, B. (1981).-"Elaboration des lipides corporels et valeurs des carcasses des ruminants".- Bull. Techn. CRZV Theix-INRA 45,43-50.

Bass, J., Butler-Hogg, B.W., Kirton, H.(1990).-"Practical methods of controlling fatness in farm animals" en "Reducing fat in meat animals".- Wood and Fisher, (Ed).- Elsevier Applied Science. Barking, England.

Bauchart, D. (1981).-"Digestion comparée des lipides chez les rumiants et les monogastriques".- Bull. Techn. C.R.Z.V. Theix INRA 46, 45-55.

Boatella, J. (1992).- "Changes in dietary fats in the Spanish Diet. Future Recommendations".-Seminar on Public Health: Changing Patterns of fat intake among Mediterranean Countries. WHO. Barcelona.

British Nutrition Foundation (1987).- "Trans fatty acids".- The reports of the British Nutrition Foundation, London.

Davis, C.L.- "Fats in animal Feeds".- University of Illinois, USA.

Doreau, M., Batisse, V., Bauchart, D. (1989).-“Appreciation de l'hydrogénation des acides gras alimentaires dans le rumen de la vache: étude méthodologique préliminaire".- Ann. Zootech. 38,139-144.

Emken, E.A., Dutton, H.J. (edited by) (1979).-"Geometrical and positional fatty acid isomers".- The American Oil Chemists' Society, Champaign, Illinois.

Enser, M. (1984).-"The Chemistry, biochemistry and nutritional importance of animal fats" en "Fats in animal nutrition".-Wiseman, (Ed).-Butterworths. London, England.

Folch, J., Lees, M., Sloane-Stanley, G.H. (1957).- "A simple method for isolation and purification of total lipids from animal tissues".- J Biol. Chem. 226, 497-509.

Girard, J.P., Bucharles, C., Gerardot, L., Denoyer, C. (1985).-" Les lipides animaux dans la filière viande".- Volume 1.- Editorial APRIA, París, France.

Gunstone, F.D., Norris, F.A. (1983).-“Lipids in Foods. Chemistry, Biochemistry and Technology".-Pergamon Press, Great Britain.
Gurr, M.I. (1984).- "Role of fats in food and nutrition"; pp. 18-22 y 122128.-Elsevier Applied Science. Barking, England.

Harfoot, C.G . (1973).- "Lipid metabolism in the rumen".- Prog. Lipid. Res. 17, $21-54$,

Hecker, A.L., Cramer, D.A., Hougham, D.F. (1975).-"Compositional and metabolic growth effects in the bovine. Muscle, subcutaneous and serum total fatty acids".- J . Food Sci. 40, 144-149.

Hernández, N., Codony, R., Rafecas, M., Boatella, J. (1991).-“Contenidos de isómeros trans de los ácidos grasos en productos cárnicos. (I) Embutidos".- Grasas y Aceites 42,143-147.

Hunter, J.E., Applewhite, T.H. (1986).- "Isomeric fatty acids in the US diet: Levels and health perspectives".- Am. J. Clin. Nutr. 44, 707-717.

Kinney, M., Cross, H.R., Smith, G.C., Smith, S.B. (1990).- "Subcellular distribution and composition of lipids in muscle and adipose tissues".-J. Food Sci. 55, 4345.

Lands, W.E.M., Blank, M.L., Nutter, L.J., Privett, O.S. (1966).-"A comparison of acyltransferase activities in-vitro, with the distribution of fatty acids in lecithins and triglycerides in-vivo"- Lipids 1, 224-229.

Lin, K.C., Marchello, M.J., Fischer, A.G. (1984).-"Determination of the amount of trans-octadecenoate and trans-9, trans-12-octadecenoate in fresh lean and fatty tissues of pork and beef".- J. Food. Sci. 49, 15211524.

Lluch, M.C., Pascual, J., Parcerisa, J., Guardiola, F., Codony, R., Rafecas, M. y Boatella, J. (1993).-"Contenidos de isómeros trans de los ácidos grasos en productos cárnicos. (II) Tejido adiposo y grasa intramuscular de cerdo".- Grasas y Aceites 44, 97-100.

Slover, H.T., Lanza, E. (1979).-"Quantitative analysis of food fatty acids by capillary gas chromatography".- J. Am. Oil Chemists' Soc. 56, 933-943.

Slover, H.T., Lanza, E., Thompson, R.Jr., Davis, C.S., Merola,G.V. (1987).-"Lipids in raw and cooked beef".- J. Food Comp. Analysis 1, 2637.

Sommerfeld, M. (1983).-"Trans unsaturated fatty acids in natural products and processed foods".- Prog. Lipid Res. 22, 221-233.

(Recibido: Mayo 1993) 